\title{
Nanophase Fe-Oxide, Fe-Sulfide, and Ilmenite in High-Ti Lunar Soil using Aberra- tion-Corrected STEM-EELS and EDS
}

\author{
K. D. Burgess ${ }^{1}$ and R. M. Stroud ${ }^{2}$ \\ ${ }^{1}$ ASEE Postdoc, Naval Research Laboratory, Washington, DC 20375 USA (kate.burgess.ctr@nrl.navy.mil) \\ ${ }^{2}$ Naval Research Laboratory, Washington, DC 20375 USA
}

Space weathering on airless bodies includes a number of processes, such as micrometeorite impacts and solar wind bombardment, leading to a variety of alteration features, including vapor deposited layers and creation of nanophase opaque minerals [1-3]. Although space weathering on a planetary scale has important implications for remote sensing measurements [3], direct laboratory observation and analysis of the individual nano-scale features is necessary for determining which processes and reactions dominate on specific airless bodies. Transmission electron microscopy (TEM) is the most commonly employed method for such studies [2]. Recent improvements in TEM and electron energy-loss spectroscopy (EELS) instrumentation and techniques provide significant increases in both the spatial and energy resolution, enabling better direct observation of nanophase iron oxides in mature lunar soil [4]. We report here results from an aberration-corrected scanning transmission microscope (STEM) study of the composition and oxidation state of soil grains from mature lunar soil 79221, sub-mature lunar soil 71501, and immature lunar soil 71061.

Samples were prepared using focused ion beam (FIB) microscopy. The regions of interest were coated with a thick carbon film $(1-2 \mu \mathrm{m})$ before ion milling to prevent damage to the grain surface by the ion beam. Electron energy loss spectroscopy (EELS) and energy dispersive x-ray spectroscopy (EDS) data were collected with PRISM, the NION UltraSTEM200 at the Naval Research Laboratory, equipped with a Gatan Enfinium ER EEL spectrometer and a Bruker SSD-EDS detector. The STEM was operated at $200 \mathrm{kV}$ and $\sim 40 \mathrm{pA}$, with a 0.1 to $0.2 \mathrm{~nm}$ probe. Spectra were collected as spectrum images (SI), with a spectrum collected for each pixel for mapping of possible variations in thickness, composition, and oxidation state. Moderate dwell time (0.01-0.5s) were used to balance signal-to-noise in low-Fe regions with beam damage effects. Fe L-edges in EEL spectra are caused by the excitation of inner shell (2p) electrons to the unoccupied 3d orbitals. EEL spectra of metallic $\mathrm{Fe}$ and $\mathrm{Fe}^{2+}$ - and $\mathrm{Fe}^{3+}$ - bearing minerals show distinct edge shapes and chemical shifts that depend on the oxidation state and the symmetry and coordination of the atom site.

FIB samples from a number of grains in each soil sample reveal a variety of micro- and nano-structures including glassy "splash” coatings, small mineral grains attached to the surface of larger grains, vapor deposits of varying composition and thickness, vesicles in rims and soil grain interiors, and a number of nanophase opaque grains of Fe-metal, Fe-oxide, Fe-sulfide, and ilmenite. Close analysis of some of the nanophase iron-rich grains shows variations in oxidation state, both in interior and rim regions.

As technique and instrument developments allow us to measure the composition and oxidation state of space weathered materials at finer scales, new complexity in nanophase opaques can be observed. The wide range in features seen in each of the soils emphasizes the importance of nano-scale observations of a large number of grains in order to gain understanding of space weathering processes, time evolution, and differences among airless bodies. The nanophases observed here show that processes leading to the oxidation of Fe, both in vapor deposited rims and grain interiors, need to be included in descriptions of 
lunar space weathering. Such processes could include loss of hydrogen during heating or movement of oxygen in the silicate matrix as new material is reduced. Additionally, optical measurements of nanophases other than metallic Fe may be needed to fully account for space weathering features seen in remote measurements.

\section{References:}

[1] Keller, L. P., and D. S. McKay (1993) Science, 261, 1305-1307.

[2] Keller, L. P., and D. S. McKay (1997) Geochim Cosmochim Acta, 61, 2331-2341.

[3] Pieters, C. M., et al. (2000) Meteor Planet Sci, 35, 1101-1107.

[4] Thompson, M. S., et al. (2015) LPSC, 46, Abstract \#2932.

[5] The authors acknowledge funding from NASA SSERVI RIS ${ }^{4} E$.
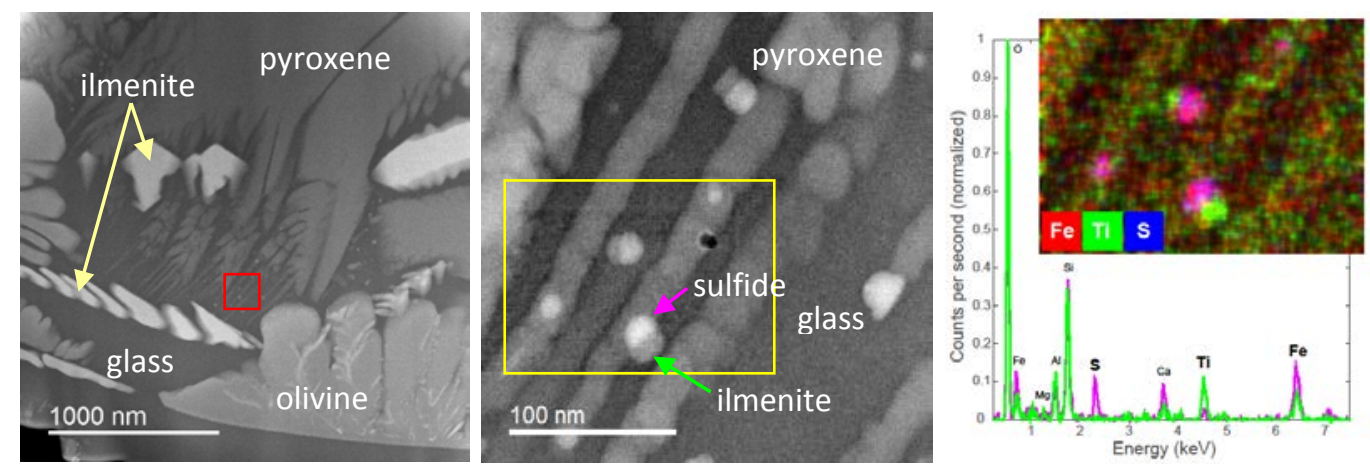

Figure 1. HAADF images of a glassy sphere showing ilmenite, olivine, pyroxene, and Fe-rich nanocrystal. EELS and EDS analysis show the nanocrystals are Fe-sulfide and ilmenite, often in close spatial association. The EDS map of Fe, Ti, and S show several particles highlighted in the selected EDS spectra.
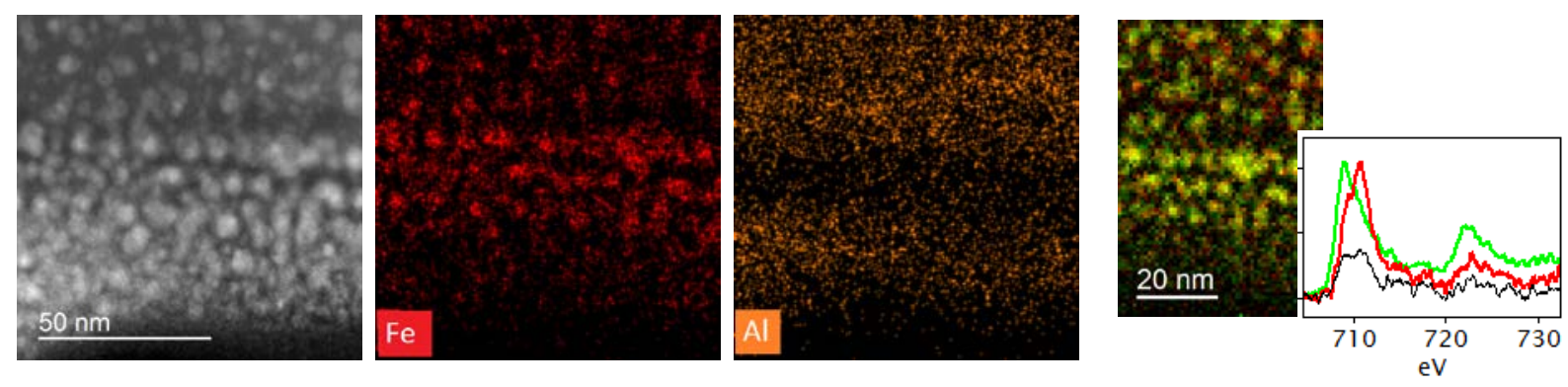

Figure 2. Multiple vapor-deposited layers with varying major element composition and nanophase Fe-rich grains are present in the vapor deposited rim of a plagioclase grain from 79221, as seen in HAADF image and Fe and Al EDS element maps. The right image shows the relative contribution of the EELS signal for oxidized (red) and reduced (green) components as shown in the spectra. The nanoparticles furthest from the surface are oxidized while the more ordered layers are metallic Fe. The amorphous material between the particles (black) has less Fe, but both oxidized and reduced states are present. Beam damage likely affects the oxidation state in the amorphous regions; however, oxidation states in crystalline regions are less affected and do not change on repeated measurements. 